\title{
A Utilização da Lógica Fuzzy Como Ferramenta Auxiliar na Detecção de Doenças Fúngicas do Tomateiro Como a Septoriose
}

\author{
Luís Fontes Moreira ${ }^{1}$, Arilson Fernando Gomes Ferreira ${ }^{1}$, \\ Gabriel Rodrigues de Arruda ${ }^{1}$, Rhyan Ximenes de Brito ${ }^{1}$ \\ ${ }^{1}$ Instituto Federal de Educação, Ciência e Tecnologia do Ceará (IFCE) \\ CE-187, s/n - Estádio, - CEP 62320-000 - Tianguá - CE - Brasil \\ \{a.fernando.ifce, luisfontes.lef, arrudagabriel97, rxbrito\}@gmail.com
}

\begin{abstract}
The fuzzy systems are appropriate for the manipulation of subjective information, as in the scenario of this research on tomato septoria. It can be used by professionals to detect the disease before it manifests itself throughout the planting. Due to the great diversity of diseases in the tomato, to detect which one affects the plant becomes too difficult, in this way the objective of this article is to discuss the use of the fuzzy logic to aid in the analysis of the plants and thus to be able to detect if it is a carrier or can to develop septoriosis. According to the results presented, the fuzzy system proved to be very useful for the aid in the detection of the disease, being evident its importance as a diagnostic tool in plants.
\end{abstract}

Resumo. Os sistemas fuzzy são apropriados para a manipulação de informações subjetivas, como no cenário desta pesquisa sobre a septoriose no tomateiro. Podendo ser utilizado por profissionais para detectar a doença antes que se manifeste em todo o plantio. Devido a grande diversidade de doenças no tomateiro, detectar qual delas afeta a planta se torna demasiadamente difícil, dessa forma o objetivo deste artigo é discutir o uso da lógica fuzzy para auxiliar na análise das plantas e assim poder detectar se a mesma é portador ou poderá desenvolver a septoriose. De acordo com os resultados apresentados o sistema fuzzy demonstrou-se bastante útil para o auxílio na detecção da doença ficando evidente sua importância como ferramenta para diagnóstico em plantas.

\section{Introdução}

A lógica fuzzy foi introduzida pelo o Dr. Lofti Zadeh da universidade da Califórnia, conhecida também como lógica difusa foi desenvolvida para propagar os valores lógicos das variáveis que podem ser quaisquer números entre 0 , equivalente ao valor falso, e 1 equivale ao valor verdadeiro. Na lógica booleana, os valores lógicos das variáveis podem ser exclusivamente 0 ou 1 [Camargos 2002].

A lógica difusa foi desdobrada para lidar com o conceito de verdade parcial de modo que se possa determinar se algo é totalmente verídico ou totalmente falso. A mudança entre o membro e o não membro está numa faixa gradual, sendo que pode ser associado um grau entre 0 , totalmente não membro, e 1 , totalmente membro [Camargos 2002]. 
Essa lógica é de suma importância, por ser plurivalente, podendo ser aplicada em diversos problemas. Nessa situação foi utilizada a lógica de fuzzy como método de auxílio de análise da doença septoriose. Para a concretização deste trabalho, fez-se imprescindível a utilização da ferramenta MatLab um programa computacional popular em todo mundo como um instrumento para a resolução de problemas científicos, matemáticos e tecnológicos.

A motivação deste trabalho está relacionada a carência de sistemas que fazem uso da lógica não tradicional, como a lógica de $f u z z y$, para auxiliar no diagnóstico de doenças do tomateiro, de modo que se possa avaliar diversas variáveis e gerar uma saída com valores linguísticos.

Este trabalho está divido em seis seções: seção 2 descreve alguns trabalhos relacionados, seção 3 apresenta um referencial teórico com os principais conceitos sobre a lógica fuzzy. A seção 4 abordará um pouco sobre a doença septoriose no tomateiro, na seção 5 será comentado sobre a Metodologia utilizada no trabalho, na seção 6 apresentase os resultados e discussões e por fim, na seção 7, é descrita a conclusão e trabalhos futuros.

\section{Trabalhos Relacionados}

Esta seção apresenta uma revisão bibliográfica sobre a utilização da lógica de fuzzy como mecanismo de apoio para diferentes tipos de diagnósticos, com diferentes abordagens e perspectivas relacionadas a lógica difusa.

[Oliveira 2015] apresenta uma abordagem para classificação automática de doenças em folhas de plantas onde a classificação se baseia na lógica fuzzy. Os resultados foram precisos e satisfatórios em relação a classificação realizada a olho nu por fitopatologistas.

[Vianna and da Cruz ] estudou a possibilidade de inclusão de um módulo de lógica nebulosa para melhorar ainda mais o desempenho do reconhecimento inteligente e de módulos automatizados para procedimentos ainda realizados de forma manual, como a definição dos limiares ideais de saída das redes.

[Alves et al. 2006] estudou o efeito do binômio temperatura e molhamento foliar no processo monocíclico da ferrugem asiática da soja, nas cultivares conquista, savana e suprema, com base em um sistema de lógica fuzzy e modelos de regressão não-linear.

[Neto and dos Santos 2016] avaliou o desenvolvimento e a produtividade do tomate híbrido em diferentes tensões de água no solo e diferentes doses de salinidade na irrigação ao longo de seu ciclo utilizando modelagem fuzzy.

Para finalizar, [Marro et al. 2010], descrevem uma pequena lista de domínios de aplicação da lógica fuzzy, no contexto da Inteligência Artificial, tais como: sistemas especialistas, sistemas multiagentes, reconhecimento de padrões, robótica, data mining, dentre outros.

\section{Fuzzy}

Por volta de 1920 um polonês chamado Jan Luasiewicz (1878-1956) utilizandose do princípio da incerteza, apresentou pela primeira vez as noções da lógica 
dos conceitos vagos onde é admissível um conjunto com valores não precisos [Aguado and Cantanhede 2010]. Porém a lógica fuzzy, também conhecida como lógica nebulosa ou difusa se tornou conhecida a partir de 1965 quando o professor Lofti Zadeh publicou o artigo Fuzzy Sets no journal Information and Control.

Para [Gomide and Gudwin 1994], a lógica fuzzy é baseada na teoria dos conjuntos fuzzy, diferindo-se dos sistemas lógicos tradicionais em suas características e seus detalhes. Nesta lógica o raciocínio exato corresponde a um caso limite do raciocínio aproximado, sendo interpretado como um processo de composição de relações nebulosas.

Segundo [Weber and Klein 2003] o sistema fuzzy é uma coleção de variáveis de entrada, uma coleção de conjuntos para a variável de saída e uma coleção de regras que associam as entradas para resultar em conjuntos para a saída.

\section{Septoriose}

Os fungos são microorganismos causadores do maior número de doenças em plantas. São os grandes vilões da tomaticultura. Cerca de $15 \%$ dos custos de produção de tomate são atribuidos ao uso de fungicidas no combate de doenças causadas por este grupo de patógenos. Os fungos de solo, particularmente, são difíceis de ser controlados, requerendo medidas integradas de manejo [Lopes and de Ávila 2005].

A (Septoria lycopersici) ocorre em todas as regiões do Brasil, sendo observada mais freqüentemente no início de período quente e chuvoso. A doença reduz a área foliar responsável pela fotossíntese, reduzindo a produtividade, além de expor os frutos a queimadura de sol, tornando-os impróprios para consumo [Lopes and de Ávila 2005].

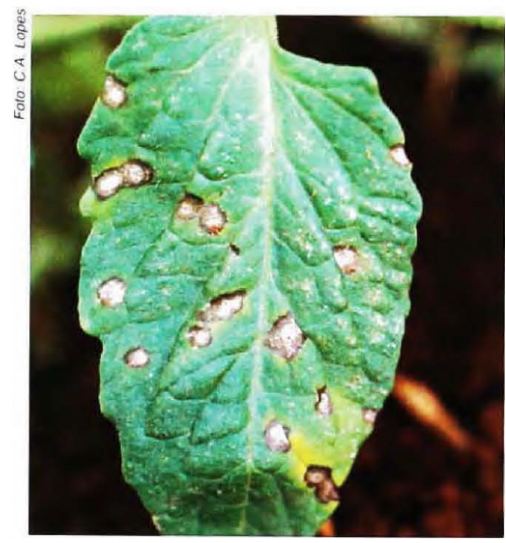

Figura 1. Folha com a doença septoriose

A septoriose é uma doença prejudicial para o tomateiro em que as folhas do tomateiro ficam cheias de manchas mais ou menos circulares e com as bordas escurecidas. É causada pelo fungo S. lycopersici Sepg, relatado pela primeira vez na Argentina, em 1882, por Spegazzini [Ferreira Junior 2016]. A doença torna-se limitante ao cultivo sob condições de chuvas constantes, o que ocorre no verão na maioria das regiões produtoras de tomate estaqueado, implicando em altos gastos com fungicidas para seu controle [Reis et al. 2006].

A prejudicialidade da septoriose reside no fato que as folhas ficam recobertas com manchas que coalescem, tomando grandes áreas da superfície foliar, fenômeno que inicia 
pelas folhas basais e se estende para o topo da planta. Normalmente, a septoriose causa desfolha na planta, expondo assim os frutos a queima pela luz solar e a maturação precoce, além de reduzir o vigor das plantas. Durante períodos de clima favorável, a doença causa desfolha quase completa, resultando em insucessos na produtividade das plantas, o que ocasiona, via de regra, grandes prejuízos econômicos [Ferreira Junior 2016].

O fungo penetra na planta através dos estômatos, e os sintomas iniciais aparecem em seis dias. Os picnídios surgem após os 14 dias da infecção. As temperaturas ótimas para ocorrência de epidemias da doença estão entre 20 a 25 graus Celsius [Ferreira Junior 2016].

\section{Metodologia}

Com o auxílio do MatLab, desenvolveu-se um sistema fuzzy para auxiliar no diagnóstico da septoriose no tomateiro. Os testes iniciais forem realizados através de dados coletados em uma cidade do interior do Ceará mais precisamente na Serra da Ibiapaba.

Foram usadas como variáveis de entrada os sintomas que as folhas do tomateiro apresentaram, como: manchas arredondadas, bordas escurecidas, centro na cor de palha, folhas inferiores, ponto preto no centro da mancha e folhas secas. A partir das combinações das variáveis gerou diversas regras de inferências, algumas dessas regras estão sendo representadas pela Figura 2.

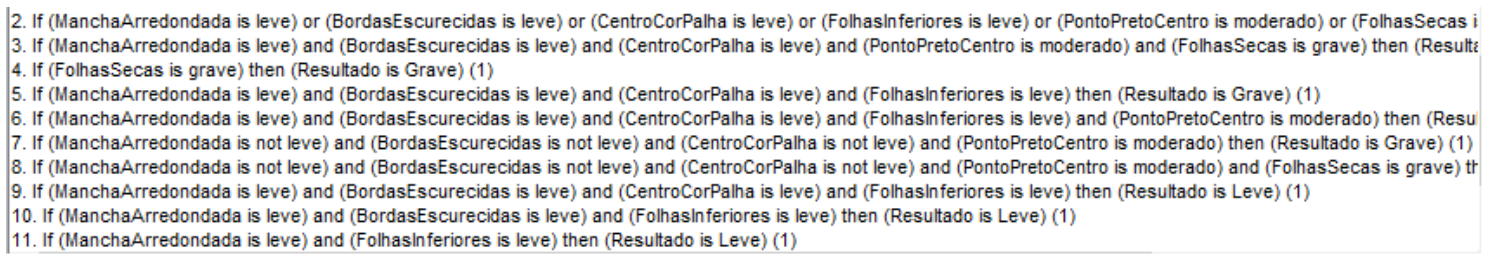

Figura 2. Regras de Inferências do Sistema Fuzzy

Esses sintomas são quantificados em uma escala de 0 a 6 , onde os valores de zero a 3.9 representam a fase leve da doença, a qual representa um nível de severidade leve da doença sendo de fácil contenção, ao atingir a escala de 4 a 5 passa para fase moderada e acima de 5 para a fase grave, onde o nível de severidade da doença está alto. O diagnóstico está quantificado na escala de 0 a 0.6 .

A Figura 3 apresenta as variáveis de entrada na ferramenta MatLab. Nesse trabalho a aplicação possui seis variáveis de entrada e uma de saída, onde as variáveis de entrada correspondem aos sintomas necessários para a identificação da doença septoriose. Vale salientar que o sintoma principal é a mancha circular que aparece nas folhas e os demais sintomas são o agravamento dessa mancha. Os sintomas utilizados como entradas no desenvolvimento da aplicação foram classificados em uma escala entre 0 e 6 , onde 0 é a menor atribuição e 6 é a maior atribuição.

\section{Resultados e Discussões}

Para demonstrar a eficácia da aplicação foram analisados diversos casos de testes através de simulações, nesta seção foram abordados 3 dos resultados gerados pelo sistema, com base no diagnóstico do Tomateiro X, Tomateiro Y e Tomateiro Z. 


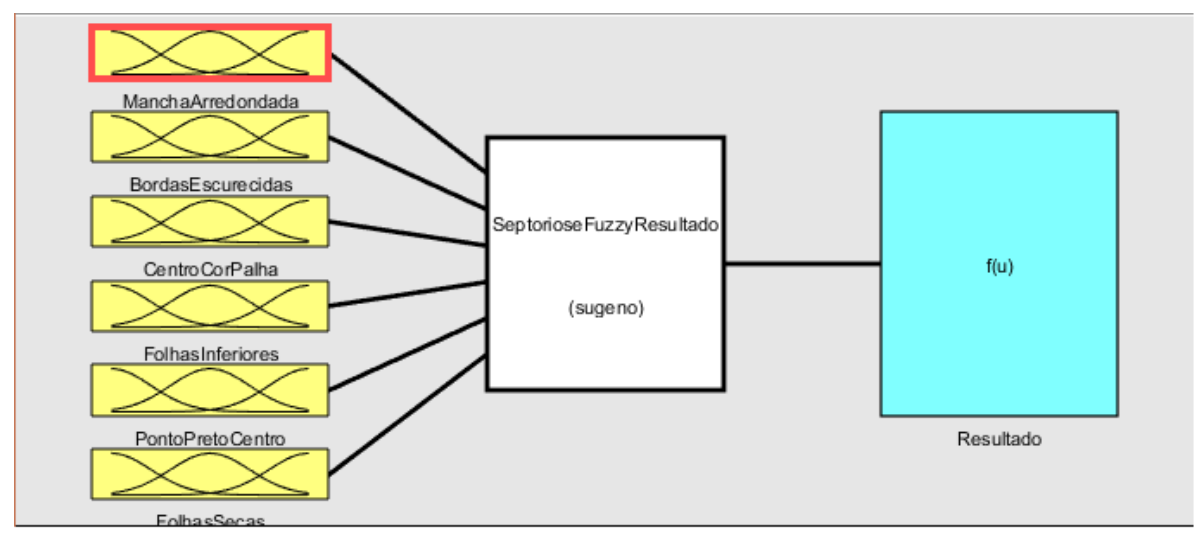

Figura 3. Representação do Sistema Fuzzy

A Figura 4 apresenta dados para a gravidade de cada sintoma e o diagnóstico obtido com a planta Tomateiro X, onde foi verificado que os sintomas pré-definidos apresentaram os seguintes resultados: manchas arredondadas com um indício de gravidade de 0.5 , considerado início da doença, bordas escurecidas com índice 1.5, também considerado leve, centro na cor palha com 0.5 , folhas inferiores com 3 , ponto preto no centro da mancha com 4.2, sendo já um sinal moderado tendendo ao estágio grave e por último folhas secas com 5.1, considerado grave. O diagnóstico obtido foi 0.21 , que seria um caso em que a doença septoriose estaria em uma fase inicial de infestação, sendo um sinal de alerta pela próximidade de uma possível epidemia.
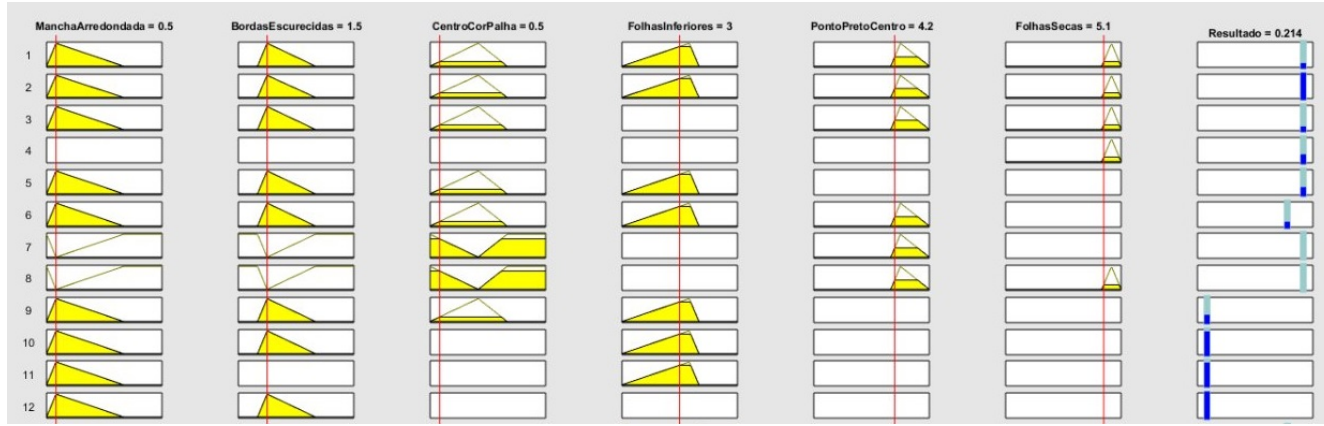

Figura 4. Diagnóstico do Tomateiro $X$

A Figura 5 também possui dados para a gravidade de cada sintoma e o diagnóstico obtido com a planta Tomateiro Y, onde foi verificado que os sintomas pré-definidos apresentaram os seguintes resultados: manchas arredondadas com um índicio de gravidade de 3.4, considerado início da doença, mas já bastante infestado, bordas escurecidas com índice 1.5, também considerado leve, centro na cor palha com 0.6 , folhas inferiores com 3 , ponto preto no centro da mancha com 4.2, sendo um sinal leve, porém bem intenso tendendo ao estágio moderado e por último folhas secas com 5.1, considerado grave. $\mathrm{O}$ diagnóstico obtido foi 0.43 , que seria um caso em que a doença septoriose estaria na fase moderada.

Por fim a Figura 6 apresenta dados para a gravidade de cada sintoma e o diagnóstico obtido com a planta Tomateiro Z, onde foi verificado que os sintomas prédefinidos apresentam os seguintes resultados: manchas arredondadas com um indício de 

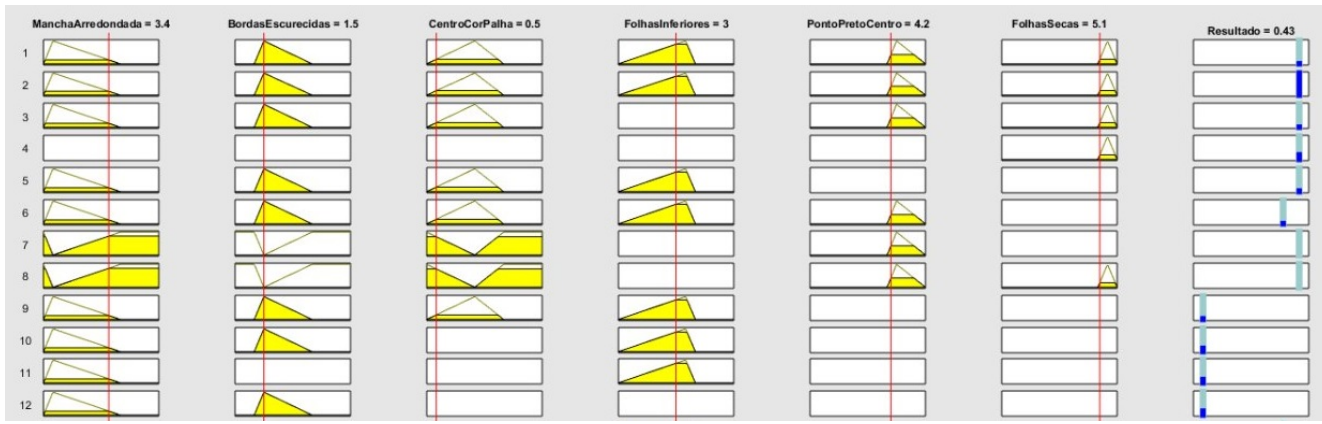

Figura 5. Diagnóstico do Tomateiro $Y$

gravidade de 5.66, considerado início da doença, mas com gravidade alta, bordas escurecidas com índice 5.3, considerado grave, centro na cor palha com 5.15, folhas inferiores com 4.58, ponto preto no centro da mancha com 5.04, sendo já um sinal tendendo ao estágio grave e por último folhas secas com 6 , considerado grave. O diagnóstico obtido foi 0.584 , que seria um caso em que a doença septoriose estaria bastante avançada.
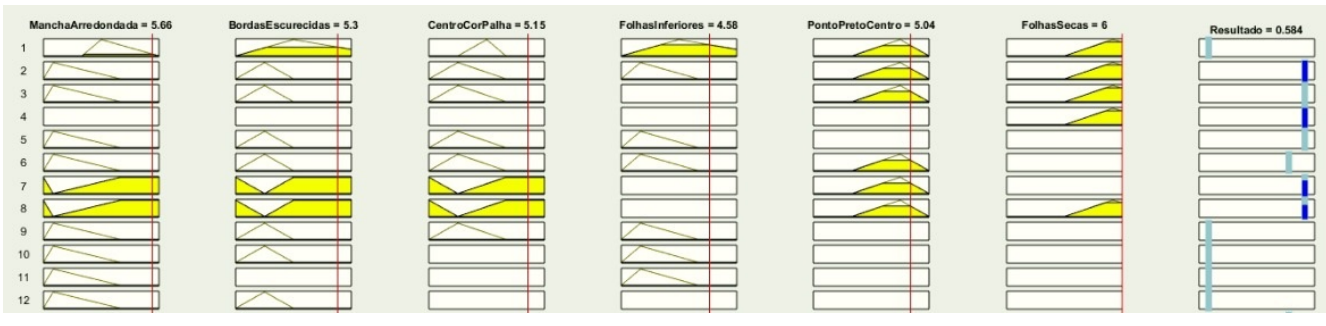

Figura 6. Diagnóstico do Tomateiro Z

\section{Conclusão e Trabalhos Futuros}

Este trabalho teve como finalidade mostrar a utilização da lógica fuzzy como mecanismo auxiliador no diagnóstico de doenças fúngicas como a septoriose que acomete o tomateiro. Para os testes foram utilizados dados fictícios. Entretanto pode ser validado com dados reais e adaptado para produzir resultados confiáveis que poderão ser utilizados por profissionais da agricultura.

O sistema poderá ser usado para auxíliar na detecção precoce da septoriose, pois ao se detectar os primeiros sitomas da doença os cuidados necessários para combater serão mais eficientes. Porém o monitoramento das áreas e épocas mais suceptíveis a infestações de doenças fúngicas com o uso da lógica nebulosa, podem ser ultilizados para garantir um manejo preventivo com a utilização de fungicidas indicados por um profissional da área.

Como trabalhos futuros sugere-se a utilização da lógica fuzzy em outros tipos de doenças fúngicas do tomateiro como o oídio ou a pinta-preta por apresentarem um grau elevado de proliferação e mortalidade das plantas do tomateiro principalmente em regiões como a da Ibiapaba no estado do Ceará.

\section{Referências}

Aguado, A. G. and Cantanhede, M. A. (2010). Lógica fuzzy. Artigo sem. 
Alves, M., POZZA, E., SILVA, F., SOUZA, P., and POZZA, A. (2006). Akutsu, m. relação de funções climáticas e bióticas com a taxa de infecção da ferrugem do cafeeiro (hemileia vastatrix berk. \& br.). 1981. 67. GEOESTATÍSTICA E SISTEMAS 'FUZZY'NA PROTEÇÃO DE PLANTAS, 45:93.

Camargos, F. L. (2002). Lógica nebulosa: uma abordagem filosófica e aplicada. Universidade Federal de Santa Catarina (UFSC).

Ferreira Junior, J. B. (2016). Eficiência de fungicidas no controle da septoriose em tomateiro industrial.

Gomide, F. A. C. and Gudwin, R. R. (1994). Modelagem, controle, sistemas e lógica fuzzy. SBA controle \& Automação, 4(3):97-115.

Lopes, C. A. and de Ávila, A. (2005). Doenças do tomateiro. Embrapa Hortaliças-Livro técnico (INFOTECA-E).

Marro, A. A., de Carvalho Souza, A. M., de Sousa Cavalcante, E. R., Bezerra, G. S., and de Oliveira Nunes, R. (2010). Lógica fuzzy: Conceitos e aplicações. Universidade Federal do Rio Grande do Norte (UFRN).

Neto, V. and dos Santos, D. (2016). Modelagem fuzzy para avaliação do desenvolvimento do tomate em tensões de água no solo e doses de salinidade na irrigação.

Oliveira, C. C. (2015). Detecção automática de doenças em folhas de plantas baseado em redes de petri e algoritmos genéticos.

Reis, A., Boiteux, L. S., and Lopes, C. A. (2006). Mancha-de-septória: doença limitante do tomateiro no período de chuvas. Embrapa Hortaliças.

Vianna, G. K. and da Cruz, S. M. S. Redes neurais artificiais aplicadas ao monitoramento inteligente de doenças e pragas em tomateiros.

Weber, L. and Klein, P. A. T. (2003). Aplicação da lógica fuzzy em software e hardware. Editora da ULBRA. 